Paper accepted : 1995 SBMO/IEEE MTT-S International Microwave and Optoelectronics Conference, Rio de Janeiro, Brazil, July 24-27, 1995.

\title{
COUPLING SINGLE-MODE FIBER TO UNIFORM AND SYMMETRICALLY TAPERED THIN-FILM WAVEGUIDE STRUCTURES USING GADOLINIUM GALLIUM GARNET
}

\author{
Jagannath Gadi, Raj Yalamanchili and Mohammad Shahid \\ Howard University \\ Department of Electrical Engineering \\ 2300,6 th Street, N.W \\ Washington, DC 20059
}

\begin{abstract}
The need for high efficiency components has grown significantly due to the expanding role of fiber optic communications for various applications. Integrated optics is in a state of metamorphosis and there are many problems awaiting solutions. One of the main problems being the lack of a simple and efficient method of coupling single-mode fibers to thin-film devices for integrated optics. In this paper, optical coupling between a single-mode fiber and a uniform and tapered thin-film waveguide is theoretically modeled and analyzed. A novel tapered structure presented in this paper is shown to produce perfect match for power transfer.
\end{abstract}

\section{INTRODUCTION}

Integrated Optics has come a long way since early 1970's. It is poised at the threshold of making a big impact in everyday world. Integrated optics has drawn into several disciplines, such as computers and microwave integrated circuit technology resulting in new fabrication technologies. The field is now in a state of flux and there are still many problems awaiting solutions. One of the main problems being the lack of a simple and efficient method of coupling optical fiber to thin-film devices for integrated optics. Although a number of connectors are available in the market, the efficiency and reproducibility are low. In this paper, a novel uniform and symmetrically tapered structure are analyzed mathematically and the results presented.

There are certain papers published by researchers in the area of thin-film couplers that are worthy of note, in that they provide the background for the development of the proposed work and point towards issues raised by shortcomings of the previous work. Louisell [I] investigated broadband bi-directional couplers in which he showed that the phase constants and coupling coefficients vary with distance along two coupled transmission lines. Ulrich [2] has shown by analysis how light can be coupled into a thin-film by means of a prism-film coupler. Akira Ihaya [3] presented a mathematical model of a thin-film optical directional coupler consisting of a three-layered deposited glass films on the substrate, with coupling occurring between the first and the third film. Wilson and Teh[4] have shown a mathematical modeling of a tapered velocity directional coupler. Nelson [5] has theoretically 
examined the coupling of single-mode optical waveguides through the use of expanding and contracting tapers. Juichi Noda et al.[6] have shown a connection between single mode fiber coupler to a Ti diffused LiNo, strip waveguide. The authors Y.Cai et al.[7]., have analyzed the coupling characteristics of a uniform structure.

\section{THEORETICAL BACKGROUND}

In this paper, we present a novel method of coupling light from a single mode fiber to two structures : Uniform and symmetrically tapered thin-film couplers. The model presented is an improved version of the uniform five layered structure presented by Y. Cai et al [7]. If a fiber is directly connected to the thin-film, as shown by Y.Cai et al [7]., it leads to a large mismatch of the field profiles at the interface and as a result, leads to loss of optical power. To overcome this optical reflection and radiation losses, they proposed a five layered structure which is shown in Figure 1. This consists of a coupling waveguide, buffer layers, thin-film layer and the substrate. To avoid mismatch of the fiber core and the coupling waveguide field profiles at the interface, the coupling waveguide dimensions are designed as $2 \mathrm{aX} 2 \mathrm{a}$, where ' $\mathrm{a}$ ' is the radius of the fiber core. Guttmann et al[8]., have shown that the field distributions of the fundamental mode of the cylindrical fiber core and the garnet material waveguide differ slightly if the refractive index differences are smaller. Solgel solution is used at the interface such that any slight refractive index mismatch between the fiber core and the coupling waveguide is minimized. The buffer layer serves the purpose of coupling optical power to the thin-film waveguide of lower refractive index. Gadolinium Gallium Garnet material was used as the thin-film material because of the properties exhibited by the material. The buffer layers and the coupling waveguide are silica doped materials. The required refractive index of buffer and coupling waveguides is obtained by doping $\mathrm{TiO}_{2}$ with $\mathrm{SiO}_{2}$

The eigen mode equations of the five layered structure developed (Figure 1 ) can be obtained by solving with $w=g=2 a$, where : $w=$ the height of the coupling waveguide, $a=$ radius of the fiber core, $n_{m}=$ refractive index of the coupling waveguide ( Garnet material ), $\mathrm{n}_{\mathrm{a}}=$ refractive index of the buffer layer, $\mathrm{n}_{\mathrm{w}}=$ refractive index of the thinfilm material, $\lambda=$ wavelength of the wave $(1.3 \mu \mathrm{m})$ and $W 1=$ height of the thin-film coupling waveguide. The eigenmode equations developed by Y.Cai et al[9].,are :

$$
\begin{aligned}
& Q_{a}=n_{a}^{2} K_{g x} \tan \left(K_{g x} \cdot W / 2\right) \\
& Q_{a}=\sqrt{(2 \pi / \lambda)^{2}}\left(n_{g g}^{2}-n_{a}^{2}\right)-K_{g x}^{2} \\
& Q_{t}=\frac{\mid K_{g y}\left\{-1+\sqrt{\left.\tan ^{2}\left(g \cdot K_{g y}\right)+1\right\}} \mid\right.}{\tan \left(g K_{g y}\right)} \\
& \left.Q_{t}=\sqrt{(2 \pi / \lambda)^{2}\left(n_{a}^{2}\right.}-n_{a}^{2}\right)-K_{g y}^{2}
\end{aligned}
$$


The parameters $k_{g x}, Q_{a}$ and $k_{g y}, Q_{t}$ are the $x$ and $y$ components of the wavenumbers. $K_{g x}$ and $K_{g y}$ represent the sinusoidal variation in the $\mathrm{x}$ and $\mathrm{y}$ directions. The propagation constant of the coupling waveguide can be determined by solving equations ( 1 to 4 ),

$\beta_{g g}^{2}=\omega^{2} \mu_{o} \in_{o} n_{g g}^{2}-K_{g x}^{2}-K_{g y}^{2}$

The propagation constant $\beta_{\mathrm{w}}$ of the thin-film waveguide is obtained by replacing $n_{\mathrm{gg}}$ with $n_{w}, w$ with $W 1$ and $\beta_{\mathrm{gg}}$ with $\beta_{\mathrm{w}}$ in equations( 1 to5). The value of $\mathrm{W} 1$ for the thin-film is so chosen such that highest power coupling occurs between the coupling waveguide and the thin-film at this value. The optimum value Wl is chosen such that $\beta_{\mathrm{gg}}=\beta_{\mathrm{W}}$ for no mismatch. The thickness of the buffer layer has a direct bearing on the power coupled from the coupling waveguide to the thin-film waveguide and an optimum value is chosen such that there is a maximum power transfer from the coupling waveguide to the thin-film waveguide. The propagation constant of the thin-film varies because of the change in value of $w$ along the $z$ direction. Therefore the mismatch. $M$ depends on the propagation constant difference given by $M(z)=\frac{\beta_{88}-\beta_{W}(z)}{2 C}$

and the maximum fraction of power transferred between the coupling waveguide and the thin-film waveguide with a fixed mismatch $M$, is given by $F^{2}=\left(1+M^{2}\right)^{-1}$

Application of the theory developed by Snyder et al[10] and Snyder [11], to the model analyzed in Figure 1. gives the power coupled into the thin-film for a uniform coupler as

$\mathbf{P}^{\prime}=\mathrm{P} \mathrm{F}^{2} \operatorname{Sin}^{2} \int_{0}^{z}(C / F) d z$

where $\mathrm{P}$ is the total power introduced, $\mathrm{C}$ the coupling coefficient between the center of the coupling waveguide and the center of the thin-film waveguide. In this paper, we present a novel uniform and symmetrically tapered structure ( Figure 2 ) where the modes of a uniform waveguide form a complete set and can propagate independently from one another, while the tapered modes are coupled together and adjust their characteristics to suit the varying transverse properties of the guiding structure as they are moving along the taper. During the process of coupling the light from the uniform coupling waveguide to the thin-film waveguide, the wave is kept in the lowest order mode. For the shape shown in this paper.( Winn and Harris [12]), the power coupled at the narrow end is given by $\mathbf{P}_{\mathrm{o}}=\mathbf{P}^{\prime}\left(1-\mathrm{F}^{2} \operatorname{Sin}^{2} \int_{0}^{z}(C / F) d z\right)$

In this paper while analyzing the theoretical model of the uniform and tapered coupler we made the assumption that the materials used are lossless and therefore there are no Fresnel's reflections at the interfaces and that there are no lossess at the interface of the fiber core and the coupling waveguide. 


\section{RESULTS AND DISCUSSION}

The material used for the analysis of uniform and tapered couplers is Gadolinium Gallium Garnet (GGG). The coupling waveguide material used is GGG. Buffer layer material is spun silica which is doped with TiO2. Thinfilm material is polymerized solgel solution of $\mathrm{SiO}_{2}$ and $\mathrm{TiO}_{2}$. The refractive index of coupling waveguide. $\mathrm{n}_{\mathrm{gg}}=$ 1.9389 @ $\lambda=1.3 \mu \mathrm{m}$. The refractive index of the buffer layers is chosen, as $n_{\mathbf{a}}=1.9340$ and the refractive index of the thin-film material, $n_{w}=1.9450$. Figure 3 shows the plot of $C$ versus $d$, where $C$ is the coupling coefficient and $d$ is the distance from the center of the coupling waveguide to the center of the thin-film. This equation given by Snyder [11], is

$$
C=\left(\frac{V \Delta}{2 \pi}\right)^{1 / 2} \frac{d}{\rho^{2}} \exp \left\{\frac{-V d^{2}}{4 \rho^{2}}\right\}
$$

The refractive index of GGG versus wavelength is shown in Figure 4. The expression used to calculate the refractive index is $n^{2}-1=\sum_{i=1}^{3} A_{i} \lambda^{2} /\left(\lambda^{2}-L_{i}^{2}\right)$

where $A_{i}$ and $L_{i}$ are the sellmeier coefficients, given by Wood and Nassau [13].

By solving the equations ( 1 to5) with $w=2 a=g$, the propagation constant $\beta_{\mathrm{g}}=9.3253804 \mu \mathrm{m}^{-1}$ was obtained.

Figure 5 shows the propagation constant for the thin-film as a function of the film thickness W1. The propagation constant of the thin-film equals the propagation constant of the coupling waveguide when the thickness $\mathrm{Wl}=0.886 \mu \mathrm{m}$. The maximum coupling power is attained at $\beta_{\mathrm{gg}}=\beta_{\mathrm{W}}=9.3253804 \mu \mathrm{m}^{-1}$. Figure 6 shows the plot of coupled power versus the propagation distance for the uniform coupler of different d's for perfectly matching constants $\beta_{g}=\beta_{w}$ at thickness of thin-film $w=0.886 \mu \mathrm{m}$. Figure 7 shows the power coupled for uniform and tapered couplers with respect to the distance $z$ and the taper starts at length $L 1=700 \mu \mathrm{m}$. with slopes $(\mathrm{K})$ of the symmetrical taper at $0.0001,0.00009$ and 0.00002 . Figure 8 shows the effect of the thickness of the thin-film and the role played by the taper in the power output.

\section{CONCLUSION}

The novel structure (Figure 2) exhibits broad-bandwidth coupling characteristics and it is easy to manufacture since it does not put serious constraints on the accuracy of the coupling length of the coupler. The taper has the distinctive advantage of confining the power within the taper such that the output stays approximately near the value of the power introduced at the start of the taper. The outstanding feature of the taper is the higher efficiency as compared to the more conventional devices. 


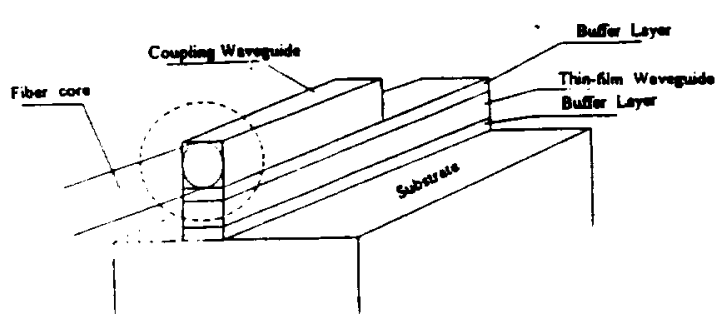

Figure 1. Schematic dlagram of Single-mode fber to thin-film waveguide using a coupling wavegulde

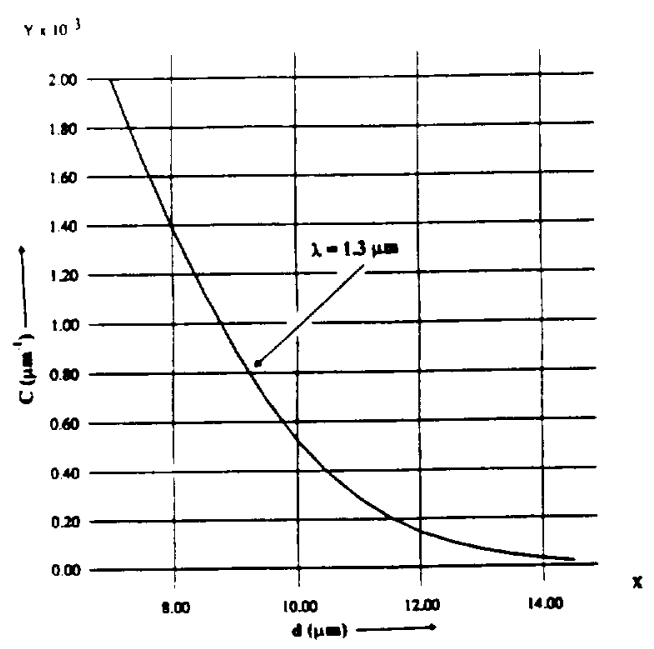

Figure 3. Coupling coeffeient versus seperation distance between center of coupling waveguide to center of thinfilm waveguide.

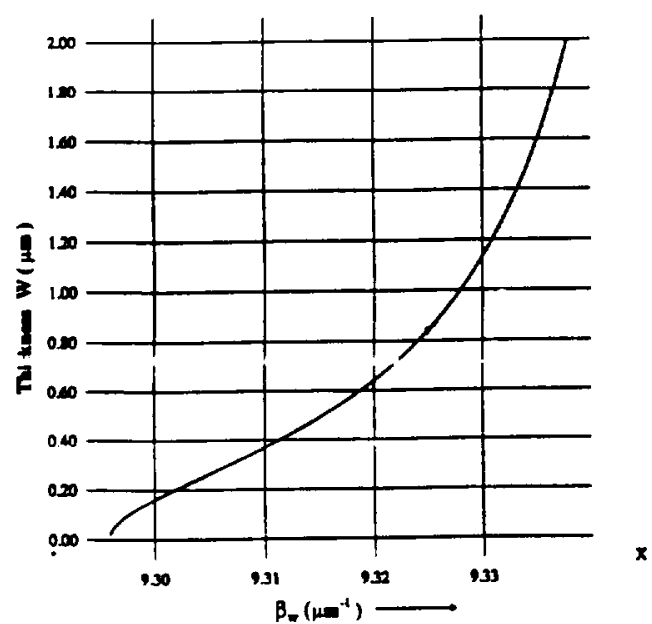

Figure 5. Propagation constants versus thickness of thinfilm.

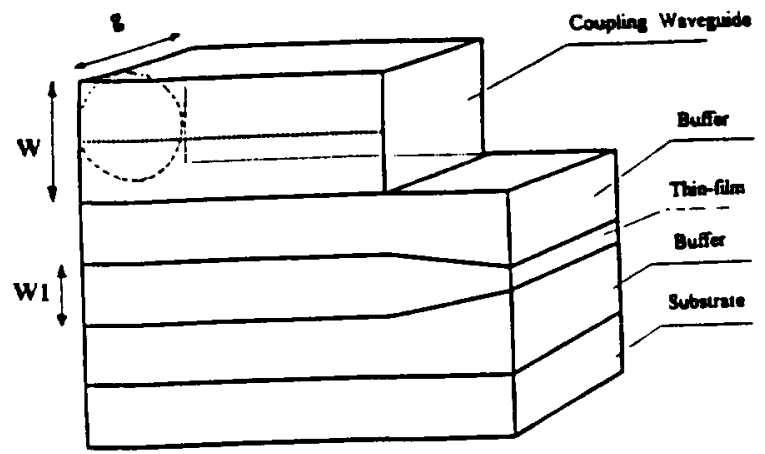

Flgure 2. Schematic diagram of the proposed novet tapered structure.

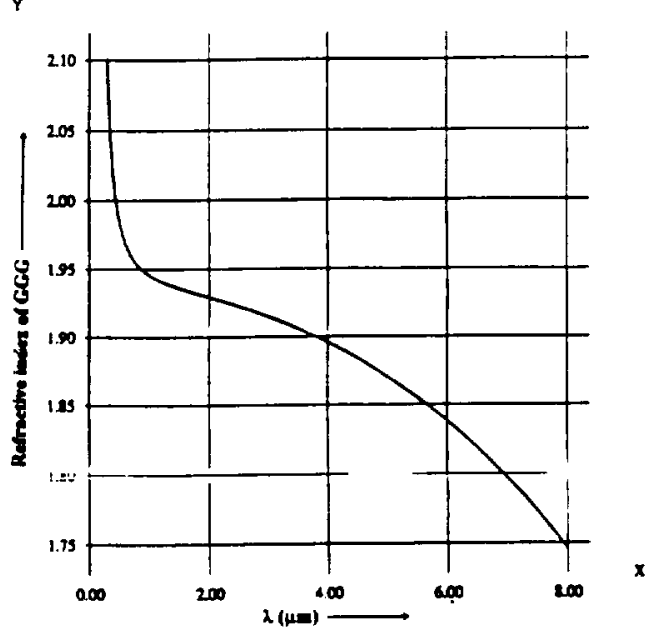

Figure 4. Refractive inder of gallium gamet as a function of wavelength.

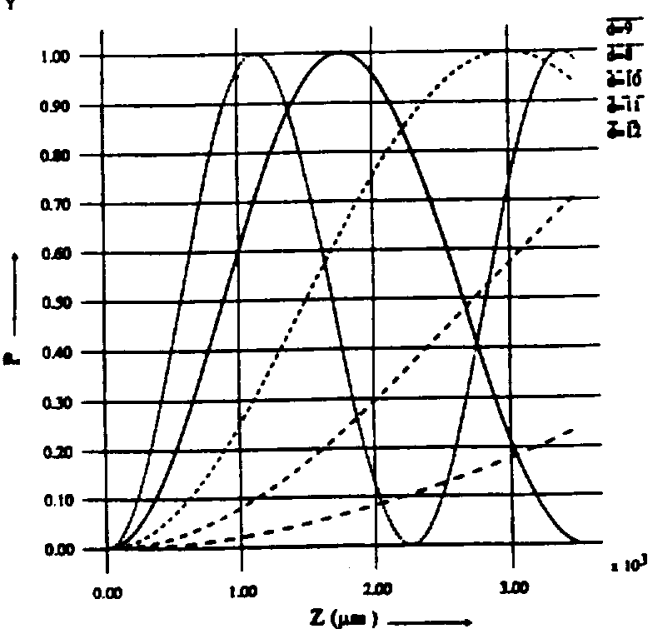

Figure 6. Plot of power coupled into thin-film versus length for vartous values of $d$. 


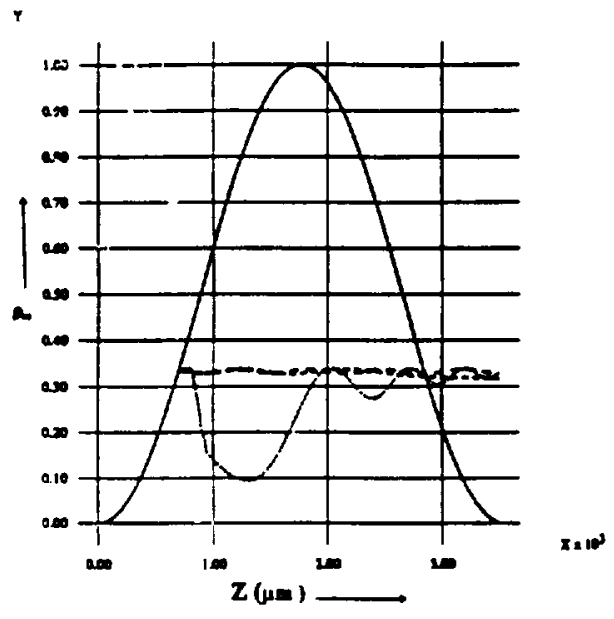

Figure 7. Comparison of uniform power and tapered power coupled into thin-film whth taper at $L 1=700 \mu \mathrm{m}$ fo $\mathrm{r}$ dirterent cases of $K$ for a thin-film thlckess of $\mathrm{W} 1=0.886 \mu \mathrm{m}$

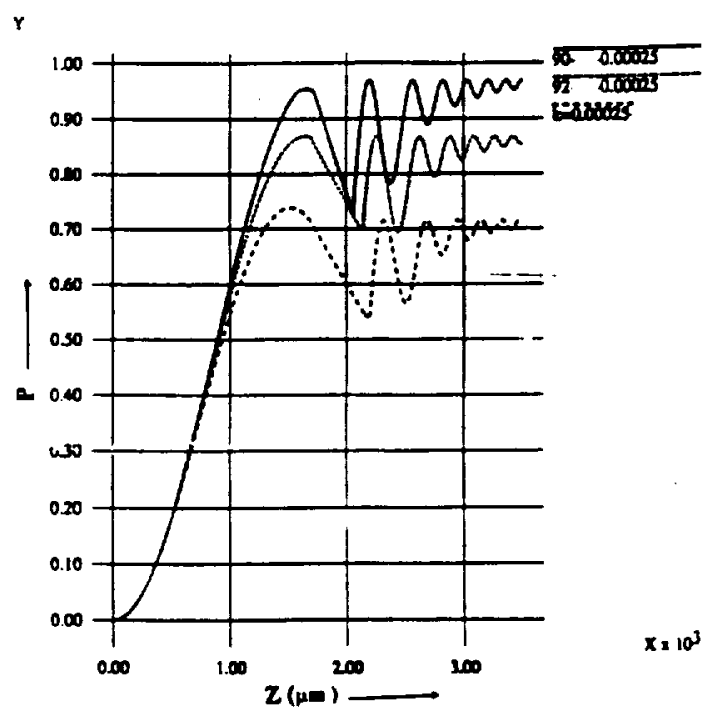

Fizure 8. Plot of coupled power wersus propagation diatance for a taper stucture with $L 1=1700 \mathrm{\mu m}$ and a slope $K=0.00025$ for difierent thiciness of thin-film.

\section{REFERENCES}

[1]. W.H.Louisell." Analysis of the single tapered mode coupler," The Bell system Technical Journal. pp 853870, July 1955.

[2]. R.Ulrich, "Theory of the prism-film coupler by plane-wave analysis", Journal of the Optical Society of America, Vol.60, No.10, pp 1337 - 1350, October 1970.

[3]. Akira Ihaya. Hirosuka Furuta. Hideki Noda. " Thin-film optical directional coupler, "Proceedings of the IEEE, pp 470-471, April 1972.

[4]. Michael G.F.Wilson and G.A. Teh." Tapered optical directional coupler," IEEE transactions on microwave theory and Techniques. Vol. MTT-23, No.1, pp 85-92, January 1975.

[5]. A.R. Nelson, “ Coupling Optical Waveguides by tapers", Applied Optics, Vol.14, No 12, December 1975.

[6]. Juichi Noda, Osamu Mikami, Makoto Minaketa, and Masaharu Fukuma," Single-mode opticalwaveguide fiber coutpler," Applied optics. Vol.17,No.13, pp 2092-2096, July 1978.

[7]. Yuanmin Cai. Tetsuya Mizumoto. and Yoshiyuki Naito, "An Effective method for coupling Single-mode fiber to Thin-film Waveguide." Vol.9. No.5, May 1991.

[\&]. J.Guttman, O.Krumpholz and E. Pfeiffer. "Optical Fiber-Stripline-Coupler”, Applied Optics. Vol.14. No.5. pp 1225-1227, May 1975.

[9]. Yuanmin Cai. Tetsuya Mizumoto. and Yoshiyuki Naito," Analysis of the coupling waveguide system." Journal of Lightwave technology, Vol.8, No. I, pp 90 - 98, January 1990.

[10]. A.W.Snyder and A. Ankiewicz." Fiber couplers composed of unequal cores," Electronics letters. Vol.22. No.23, pp 1237-1238. November 1986

[11]. Snyder and Love, "Optical Waveguide Theory", Chapman, New York, 1988.

[12]. Robert K. Winn and Jay H. Harris. " Coupling from multimode to single-mode linear waveguides using Horn-shaped structures," IEEE Transactions on Microwave Theory and Techniques, Vol. MTT-23. No. 1. January 1975.

[13]. Darvin L.Wood and Kurt Nassau . "Optical properties of Gadolinium Gallium Garnet", Applied Optics. Vol. 29, No.5. September 1990. 